\title{
ON THE TAIL BEHAVIOR OF SUMS OF DEPENDENT RISKS
}

\author{
BY
}

\section{Philippe Barbe, Anne-Laure Fougères and Christian Genest}

\begin{abstract}
The tail behavior of sums of dependent risks was considered by Wüthrich (2003) and by Alink et al. $(2004,2005)$ in the case where the variables are exchangeable and connected through an Archimedean copula model. It is shown here how their result can be extended to a broader class of dependence structures using multivariate extreme-value theory. An explicit form is given for the asymptotic probability of extremal events, and the behavior of the latter is studied as a function of the indices of regular variation of both the copula and the common distribution of the risks.
\end{abstract}

\section{KEYWORDS}

Archimedean copula; extremal behavior; multivariate regular variation; Pickands' representation.

\section{INTRODUCTION}

Consider a homogeneous portfolio of $d \geq 2$ insurance contracts, whose claim amounts over a given period are treated as continuous positive random variables $X_{1}, \ldots, X_{d}$ with common cumulative distribution function $F$ and associated survivor function $\bar{F}$. Let $S=X_{1}+\cdots+X_{d}$ denote the aggregate claim. Following Wüthrich (2003) and Alink et al. (2004, 2005), this paper considers the behavior of $\mathrm{P}(S>t)$ for large values of $t$ when the claims exhibit extreme-value behavior and are dependent. As shown by these authors, the problem is of relevance to risk managers and actuaries who wish to estimate, e.g., the Value-at-Risk (VaR) associated with $S$.

By way of introduction, suppose that each $X_{i}$ is regularly varying with index $-\beta<0$, i.e.,

$$
X_{i} \in \mathrm{RV}(-\beta) \Leftrightarrow \lim _{t \rightarrow \infty} \frac{\mathrm{P}\left(X_{i}>x t\right)}{\mathrm{P}\left(X_{i}>t\right)}=\frac{1}{x^{\beta}}, x>0 .
$$

This condition, also denoted $\bar{F} \in \mathrm{RV}(-\beta)$, is equivalent to saying that the $X_{i}$ belong to the domain of attraction of the Fréchet family of extreme-value 
distributions. It is verified, e.g., when the $X_{i}$ are Pareto random variables with $\bar{F}(t)=\mathrm{P}\left(X_{i}>t\right)=(\theta / t)^{\beta}$ for all $t \geq \theta>0$.

When the $X_{i}$ are mutually independent, it is well known (see, e.g., Feller 1971, p. 279) that

$$
\lim _{t \rightarrow \infty} \frac{\mathrm{P}(S>x t)}{\mathrm{P}\left(X_{i}>t\right)}=\frac{\Delta}{x^{\beta}}
$$

with $\Delta=d$. At the other extreme, suppose that the $X_{i}$ are comonotonic, i.e., $X_{1}=\cdots=X_{d}$ almost surely. In that case, $\mathrm{P}(S>x t)=\mathrm{P}\left(X_{i}>x t / d\right)$ and hence relation (2) holds with $\Delta=d^{\beta}$.

More generally, it is of interest to determine the constant $\Delta>0$ for a vector $X=\left(X_{1}, \ldots, X_{d}\right)$ with arbitrary dependence structure. The latter can be represented alternatively in the form

$$
\mathrm{P}\left(X_{1} \leq t_{1}, \ldots, X_{d} \leq t_{d}\right)=C\left\{F\left(t_{1}\right), \ldots, F\left(t_{d}\right)\right\},
$$

or

$$
\mathrm{P}\left(X_{1}>t_{1}, \ldots, X_{d}>t_{d}\right)=D\left\{\bar{F}\left(t_{1}\right), \ldots, \bar{F}\left(t_{d}\right)\right\}
$$

for all $t_{1}, \ldots, t_{d} \in \mathbb{R}$ in terms of unique copulas $C$ and $D$ related to one another via the Möbius decomposition formula, viz.

$$
C\left(u_{1}, \ldots, u_{d}\right)=\sum_{I \subset\{1, . ., d\}}(-1)^{|I|} D\left(1-u_{I}\right),
$$

in which $u_{I}$ is a $d$-variate vector whose $i$ th component equals $u_{i}$ or 0 according as $i \in I$ or not.

In their papers, Wüthrich (2003) and Alink et al. $(2004,2005)$ consider this question in the special case where $D$ is Archimedean, which means (see, e.g., Genest and MacKay 1986 or Nelsen 1999, Chapter 4) that there exists a function $\phi:[0,1] \rightarrow[0, \infty]$ with $\phi(1)=0$ such that for all $u_{1}, \ldots, u_{d} \in(0,1]$,

$$
D\left(u_{1}, \ldots, u_{d}\right)=\phi^{-1}\left\{\phi\left(u_{1}\right)+\cdots+\phi\left(u_{d}\right)\right\} .
$$

In order that $D$ be a distribution function, it is necessary and sufficient that $(-1)^{i} d^{i} \phi^{-1}(t) / d t^{i}>0$ for all $i \in\{1, \ldots, d\}$; see, e.g., Joe (1997, p. 109). Refer to Frees and Valdez (1998) or Cherubini et al. (2004) for applications of Archimedean copula models in actuarial science and finance.

Expressions for $\Delta$ are derived by Wüthrich (2003) and Alink et al. (2004, 2005) under conditions which amount to the assumption that $\phi(1 / t) \in \operatorname{RV}(\alpha)$ and the common margin $F$ belongs to the Fréchet domain of attraction. As they show, the constant $\Delta$ depends on $F$ and $\phi$ only through $\alpha$ and $\beta$. They reach similar conclusions when the $X_{i}$ belong to the domain of attraction of the Gumbel and Weibull extreme-value families. 
It will be shown here that the analysis of Wüthrich (2003) and Alink et al. (2004, 2005) extends well beyond Archimedean dependence structures, and that the simple formula they derive for $\Delta$ characterizes a well defined quantity that arises naturally in multivariate extreme-value theory. Specifically, it will follow from results of de Haan and Resnick (1977) that so long as the joint distribution of the $X_{i}$ exhibits multivariate regular variation of index $-\beta$, one can write

$$
\Delta=\int_{\mathbb{S}_{d-1}}\left(w_{1}^{1 / \beta}+\cdots+w_{d}^{1 / \beta}\right)^{\beta} H(d w)
$$

in terms of a so-called spectral measure $H$ defined on the set

$$
\mathbb{S}_{d-1}=\left\{w=\left(w_{1}, \ldots, w_{d}\right): w_{1}+\cdots+w_{d}=1\right\} \subset[0,1]^{d} .
$$

The developments leading to this result are summarized in Section 2, along with means of computing $H$ from $C$. Various examples are given in Section 3. The connection between the present results and those of Wüthrich (2003) and Alink et al. $(2004,2005)$ is briefly examined in Section 4, and a few concluding remarks are made in Section 5.

\section{DERIVATION AND PROPERTIES OF $\Delta$}

As it turns out, formula (7) can be deduced from classical developments in multivariate extreme-value theory. The result is possibly not new, but yields interesting insights. Minimum background information required to understand it is given below. Additional details about the general theory may be found, e.g., in Resnick $(1987,2004)$ or Mikosch (2004). In the sequel, operations on vectors are understood to be made componentwise.

Consider a vector $X=\left(X_{1}, \ldots, X_{d}\right)$ of positive random variables with common cumulative marginal distribution, $F$. Following Wüthrich (2003) and Alink et al. $(2004,2005)$, continuity of $F$ is assumed to insure the uniqueness of the copula associated with $X$. Contrary to these authors, however, the nature of the association among the $X_{i}$ is not specified at the outset.

For brevity, only the case where $F$ belongs to the domain of attraction of the Fréchet extreme-value family is treated in detail. It is thus assumed henceforth that the joint extremal behavior of the risks is such that $X$ is multivariate regularly varying of index $-\beta<0$. This condition, denoted $X \in \mathrm{MRV}_{d}(-\beta)$, implies that each of its components verifies (1).

\subsection{Definition of $\Delta$ and connection to (2)}

As illustrated, e.g., by Theorem 1 of Resnick (2004), the condition $X \in \operatorname{MRV}_{d}(-\beta)$ can be defined in many ways. In particular, it may be taken to mean that there 
exists a probability measure $\mu$, a function $b:(0, \infty) \rightarrow(0, \infty)$ that is unbounded as $t \rightarrow \infty$, and a scalar $\Delta=\Delta(b)>0$ such that

$$
\lim _{t \rightarrow \infty} t \mathrm{P}\left(|X|>x b(t), \frac{X}{|X|} \in A\right)=\frac{\Delta}{x^{\beta}} \mu(A),
$$

for any Borel set $A \subset\left\{x \in \mathbb{R}_{+}^{d}:|x|=1\right\}$.

In this definition, $|X|$ could be any fixed norm on $\mathbb{R}^{d}$, but the natural choice here is $|X|=X_{1}+\cdots+X_{d}=S$, so that $\mu$ is concentrated on $\mathbb{S}_{d-1}$. Remark 1 of Resnick (2004) further implies that no generality is lost by taking $b$ to be the generalized inverse of $g=1 / \bar{F}$, viz.

$$
b(t)= \begin{cases}\inf \{x \in \mathbb{R}: g(x) \geq t\} & \text { if } t \geq 1, \\ 0 & \text { if } t \in[0,1] .\end{cases}
$$

With this particular choice of $b$, one gets $\mathrm{P}\left\{X_{i}>b(t)\right\}=1 / t$, and the corresponding constant $\Delta$ plays the same role as in (2), since

$$
\lim _{t \rightarrow \infty} \frac{\mathrm{P}(S>x t)}{\mathrm{P}\left(X_{i}>t\right)}=\lim _{t \rightarrow \infty} \frac{\mathrm{P}\{S>x b(t)\}}{\mathrm{P}\left\{X_{i}>b(t)\right\}}=\lim _{t \rightarrow \infty} t \mathrm{P}\{S>x b(t)\}=\frac{\Delta}{x^{\beta}},
$$

where the last equality is obtained upon taking $A=\mathbb{S}_{d-1}$ in (8).

\subsection{A second expression for $\Delta$}

Introduce $Y=g(X)=\left(g\left(X_{1}\right), \ldots, g\left(X_{d}\right)\right)$ and observe that $X$ and $Y$ share the same copula, since the latter is invariant by monotone increasing transformations of the marginals. Given that the vectors $X$ and $b(Y)$ also have the same distribution, the three events in the following chain of equivalence must have the same probability:

$$
S>t \Leftrightarrow \sum_{i=1}^{d} b\left(Y_{i}\right)>t \Leftrightarrow \frac{Y}{g(t)} \in \Omega(t)=\left\{\left(z_{1}, \ldots, z_{d}\right) \in[0, \infty)^{d}: \sum_{i=1}^{d} b\left\{g(t) z_{i}\right\}>t\right\} .
$$

Further note that by Proposition 0.8 on p. 22 of Resnick (1987),

$$
\bar{F} \in \mathrm{RV}(-\beta) \Leftrightarrow g=1 / \bar{F} \in \mathrm{RV}(\beta) \Leftrightarrow b=g^{-1} \in \mathrm{RV}(1 / \beta),
$$

whence $b\{g(t) z\} / t \rightarrow z^{1 / \beta}$ as $t \rightarrow \infty$. Therefore,

$$
\lim _{t \rightarrow \infty} \Omega(t)=\Omega=\left\{\left(z_{1}, \ldots, z_{d}\right) \in[0, \infty)^{d}: z_{1}^{1 / \beta}+\cdots+z_{d}^{1 / \beta}>1\right\} .
$$

Now it is a simple matter to check that $X \in \operatorname{MRV}_{d}(-\beta) \Leftrightarrow Y \in \operatorname{MRV}_{d}(-1)$, so that in the light of Theorem 1 in Resnick (2004), there exists a (non-identically zero) Radon measure $v$ on the punctured space $\mathbb{E}=[0, \infty]^{d} \backslash\{0\}$ such that 


$$
\lim _{t \rightarrow \infty} t \mathrm{P}(Y / t \in B)=v(B)
$$

for any relatively compact set $B \subset \mathbb{E}$ whose boundary is $v$-negligible. The term "Radon measure" here simply means that $v(B)<\infty$ whenever $B$ is compact. Letting $x=1$ in (9), one may thus conclude that

$$
\Delta=\lim _{t \rightarrow \infty} \frac{\mathrm{P}(S>t)}{\mathrm{P}\left(X_{i}>t\right)}=\lim _{t \rightarrow \infty} g(t) \mathrm{P}\left\{\frac{Y}{g(t)} \in \Omega(t)\right\}=\lim _{t \rightarrow \infty} t \mathrm{P}(Y / t \in \Omega)=v(\Omega) .
$$

\subsection{Derivation of (7) and computation of $H$}

Formula (7) is a simple consequence of (10), given the spectral decomposition of $v$. For, it follows, e.g., from Theorem 1 in Resnick (2004) that there exists a positive measure $H$ on $\mathbb{S}_{d-1}$ such that

$$
v \circ T^{-1}(d r, d w)=r^{-2} d r H(d w),
$$

where $T: \mathbb{R}_{+}^{d} \backslash\{0\} \rightarrow(0, \infty) \times \mathbb{S}_{d-1}$ denotes the continuous, bijective polar-coordinate transformation

$$
x=\left(x_{1}, \ldots, x_{d}\right) \mapsto T(x)=\left(|x|, \frac{x}{|x|}\right) .
$$

Note that

$$
\int w_{i} H(d w)=1, \quad i \in\{1, \ldots, d\}
$$

and that when $|X|=X_{1}+\cdots+X_{d}$, one has also $H\left(\mathbb{S}_{d-1}\right)=d$; see, e.g., Beirlant et al. (2004, p. 260). The key observation is that

$$
T(\Omega)=\Omega^{*}=\left\{(r, w) \in[0, \infty) \times \mathbb{S}_{d-1}: r>\left(w_{1}^{1 / \beta}+\cdots+w_{d}^{1 / \beta}\right)^{-\beta}\right\}
$$

and hence from (10) that

$$
\Delta=v \circ T^{-1}\left(\Omega^{*}\right)=\int_{\Omega^{*}} r^{-2} d r H(d w)=\int_{\mathbb{S}_{d-1}}\left(w_{1}^{1 / \beta}+\cdots+w_{d}^{1 / \beta}\right)^{\beta} H(d w) .
$$

Now this conclusion, already given in (7), would be of little practical use, except for the fact that $v$ and $H$ are connected via the representation theorem of Pickands (1981), viz.

$$
v\left([0, x]^{c}\right)=v\left(\mathbb{E} \backslash \prod_{i=1}^{d}\left[0, x_{i}\right]\right)=\int_{\mathbb{S}_{d-1}} \max \left(\frac{w_{1}}{x_{1}}, \ldots, \frac{w_{d}}{x_{d}}\right) H(d w) .
$$

In particular, suppose that the probability measure $H / d$ is absolutely continuous on the interior of $\mathbb{S}_{d-1}$, and let $h / d$ denote the associated density with respect to Lebesgue measure. It follows from Theorem 1 of Coles and Tawn (1991) that 


$$
h(w)=-\frac{\partial^{d}}{\partial w_{1} \cdots \partial w_{d}} v\left([0, w]^{c}\right), w \in \operatorname{int}\left(\mathbb{S}_{d-1}\right) .
$$

An alternative proof of this result is given by Beirlant et al. (2004, p. 263). The same authors (ibid., p. 257) point out that the left-hand side can be derived from the copula associated with $X$ or $Y$ through

$$
v\left([0, x]^{c}\right)=\lim _{t \rightarrow \infty}\left\{1-C\left(1-\frac{1}{x t}\right)\right\}, x \in[0, \infty)^{d} \backslash\{0\} .
$$

The latter result is due to Huang (1992) who calls $x \mapsto v\left([0,1 / x]^{c}\right)$ the "stable tail dependence function." Its computation is particularly easy when the domain of attraction of $C$ is known. For, if $C^{*}$ is the copula of the extreme-value distribution associated with $X$, then

$$
v\left([0, x]^{c}\right)=-\log \left\{C^{*}\left(e^{-1 / x}\right)\right\} .
$$

The above developments may be summarized formally as follows.

Proposition 2.1. Assume that $X \in \mathrm{MRV}_{d}(-\beta)$ belongs to the domain of attraction of an extreme value distribution with copula $C^{*}$. Then (2) holds with $\Delta$ expressed by (7) in terms of the spectral measure $H$. The latter can be determined explicitly either from (13) and (16), or from (14) and (16).

\subsection{Basic properties of $\Delta$}

Expression (7) shows how $\Delta$ depends explicitly on $\beta$ and $H$, which characterize the marginal and joint tail behavior of the $X_{i}$, respectively. Simple consequences of this fact are stated next.

Proposition 2.2. For given $H, \Delta=\Delta(\beta)$ is an increasing function such that $\Delta(1)=d$,

$$
\begin{aligned}
& \lim _{\beta \rightarrow 0} \Delta(\beta)=\int_{\mathbb{S}_{d-1}} \max \left(w_{1}, \ldots, w_{d}\right) H(d w) \text { and } \\
& \lim _{\beta \rightarrow \infty} \frac{\Delta(\beta)}{d^{\beta}}=\int_{\mathbb{S}_{d-1}}\left(w_{1} \cdots w_{d}\right)^{1 / d} H(d w) .
\end{aligned}
$$

In the light of (13) and (16), one has also $t\{1-C(1-1 / t)\} \rightarrow-\log \left\{C^{*}(1 / e)\right\}=\Delta(0)$ as $t \rightarrow \infty$.

Proof. That $\Delta(1)=d$ stems from (11). It is also a simple matter to check that for fixed $w \in \mathbb{S}_{d-1}, \lambda(\beta)=\left(w_{1}^{1 / \beta}+\cdots+w_{d}^{1 / \beta}\right)^{\beta}$ is an increasing function of $\beta$, whence the same holds for $\Delta$ upon integration. Indeed,

$$
\lambda^{\prime}(\beta)=\left(\sum_{i=1}^{d} w_{i}^{1 / \beta}\right)^{\beta-1}\left\{\left(\sum_{i=1}^{d} w_{i}^{1 / \beta}\right) \log \left(\sum_{i=1}^{d} w_{i}^{1 / \beta}\right)-\sum_{i=1}^{d} w_{i}^{1 / \beta} \log \left(w_{i}^{1 / \beta}\right)\right\} \geq 0
$$


if and only if the expression in curly brackets is positive, i.e., whenever $x_{1}$ $\log \left(x_{1}\right)+\cdots+x_{d} \log \left(x_{d}\right) \leq x \log (x)$ for arbitrary $x_{1}, \ldots, x_{d} \in(0,1)$ with $x=x_{1}+$ $\cdots+x_{d}$. The latter inequality trivially holds. As for the limiting cases, they arise from the fact that $\lambda(\beta) / d^{\beta} \rightarrow \max \left(w_{1}, \ldots, w_{d}\right)$ or $\left(w_{1} \cdots w_{d}\right)^{1 / d}$ according as $\beta \rightarrow 0$ or $\beta \rightarrow \infty$, respectively.

\section{EXAMPLES}

The computation of $\Delta$ from Proposition 2.1 is illustrated here in four simple cases.

\subsection{Asymptotic independence between the $X_{i}$}

When $X \in \operatorname{MRV}_{d}(-\beta)$ belongs to the domain of attraction of independence, one gets $v\left([0, x]^{c}\right)=1 / x_{1}+\cdots+1 / x_{d}$. In the light of Corollary 5.25 on p. 292 of Resnick (1987), $H$ then puts unit point mass on each of the vertices of $\mathbb{S}_{d-1}$, and hence $\Delta=d$ as it should be. This occurs, e.g., when the copula $C$ in (3) is differentiable at $(1, \ldots, 1)$. The latter happens in particular for the multi-parameter extension of the Farlie-Gumbel-Morgenstern copula defined by

$$
C\left(u_{1}, \ldots, u_{d}\right)=\prod_{i=1}^{d} u_{i}\left\{1+\sum_{k=2}^{d} \sum_{1 \leq j_{1}<\ldots<j_{k} \leq d} \theta_{j_{1}, \ldots, j_{k}} \prod_{\ell=1}^{k}\left(1-u_{j_{\ell}}\right)\right\}
$$

with

$$
1+\sum_{k=1}^{d} \sum_{1 \leq j_{1}<\ldots<j_{k} \leq d} \theta_{j_{1}, \ldots, j_{k}} \prod_{\ell=1}^{k} \varepsilon_{j_{\ell}} \geq 0
$$

for any choice of $\varepsilon_{1}, \ldots, \varepsilon_{d}$ in $\{-1,1\}$.

\subsection{Asymptotic comonotonicity}

When $X \in \operatorname{MRV}_{d}(-\beta)$ belongs to the domain of attraction of the Fréchet upper bound distribution whose copula is $C\left(u_{1}, \ldots, u_{d}\right)=\min \left(u_{1}, \ldots, u_{d}\right)$, one gets $v\left([0, x]^{c}\right)=\max (1 / x)$. In this case, Proposition 5.26 on p. 294 of Resnick (1987) states that $H$ puts a single mass of size $d$ at the midpoint $(1 / d, \ldots, 1 / d)$ $\in \mathbb{S}_{d-1}$. Consequently, $\Delta=d^{\beta}$, as mentioned in the Introduction.

\subsection{Extreme-value copulas in the case $d=2$}

When $d=2$, Pickands (1981) pointed out that the extreme-value attractor $C^{*}$ can be written in the form 


$$
C^{*}\left(u_{1}, u_{2}\right)=\exp \left[\log \left(u_{1} u_{2}\right) A\left\{\frac{\log \left(u_{1}\right)}{\log \left(u_{1} u_{2}\right)}\right\}\right]
$$

in terms of a dependence function $A:[0,1] \rightarrow[1 / 2,1]$ that is both convex and such that $\max (t, 1-t) \leq A(t) \leq 1$ for all $t \in[0,1]$. If $X \in \operatorname{MRV}_{2}(-\beta)$, it is then immediate from (16) that

$$
v\left([0, x]^{c}\right)=\frac{x_{1}+x_{2}}{x_{1} x_{2}} A\left(\frac{x_{2}}{x_{1}+x_{2}}\right) .
$$

Since the simplex $\mathbb{S}_{1}=\{(w, 1-w): w \in[0,1]\}$ can be identified with the unit interval, here $H$ may be assimilated to a positive measure on $[0,1]$. Let $A^{\prime}$ denote the right derivative at every $w \in[0,1)$ and write $A^{\prime}(1)=\lim _{w \rightarrow 1} A^{\prime}(w)$. Following Beirlant et al. (2004, pp. 268-269), one then has

$$
H([0, w])= \begin{cases}1+A^{\prime}(w) & \text { if } w \in[0,1), \\ 2 & \text { if } w=1 .\end{cases}
$$

This implies that $H$ has point masses at 0 and 1, viz.

$$
H(\{0\})=1+A^{\prime}(0), H(\{1\})=1-A^{\prime}(1) .
$$

If $A$ happens to be twice differentiable on $(0,1)$, as in the mixed model of Tawn (1988), one finds

$$
\Delta=2+A^{\prime}(0)-A^{\prime}(1)+\int_{0}^{1}\left\{w^{1 / \beta}+(1-w)^{1 / \beta}\right\}^{\beta} A^{\prime \prime}(w) d w .
$$

As an illustration of Proposition 2.2, note that $\Delta(0)=2 A(1 / 2)$ when $d=2$ and $X$ has an extreme-value copula. A similar result holds also for the class of bivariate Archimax copulas introduced by Capéraà et al. (2000).

\subsection{Archimedean copulas}

Let $X \in \mathrm{MRV}_{d}(-\beta)$ and suppose that its joint distribution is specified by (3) and (6), where $\phi(1-1 / t) \in \mathrm{RV}(-\alpha)$ for some $\alpha>1$. As implied by the work of Genest and Rivest (1989), the copula $C^{*}=C_{\alpha}^{*}$ associated with the attractor is then the Gumbel-Hougaard distribution with parameter $\alpha$, viz.

$$
C_{\alpha}^{*}\left(u_{1}, \ldots, u_{d}\right)=\exp \left\{-\left(\sum_{i=1}^{d}\left|\log u_{i}\right|^{\alpha}\right)^{1 / \alpha}\right\} .
$$

The value of $\alpha$ can easily be computed, e.g., for the copula families numbered (4.2. $x$ ) for $x=1, \ldots, 22$ in Table 1 of Nelsen (1999). One gets $\alpha=\infty$ for families 
$x=18,19$, and in examples $x \in\{2,4,6,12,14,15,21\}, \alpha$ coincides with the dependence parameter noted $\rho$ by Nelsen (1999). In the remaining examples, $\alpha=1$.

When $C$ is Archimedean with generator $\phi(1-1 / t) \in \mathrm{RV}(-\alpha)$, it follows from (17) that

$$
v_{\alpha}\left([0, x]^{c}\right)=\left(\sum_{i=1}^{d} x_{i}^{-\alpha}\right)^{1 / \alpha} .
$$

In the light of (12), one can then conclude that

$$
\Delta=\Delta_{1}(\alpha, \beta)=\int_{\mathbb{S}_{d-1}}\left(\sum_{i=1}^{d} w_{i}^{1 / \beta}\right)^{\beta} H_{\alpha}(d w)=\int_{\mathbb{S}_{d-1}}\left(\sum_{i=1}^{d} w_{i}^{1 / \beta}\right)^{\beta} h_{\alpha}(w) d w .
$$

The last identity is justified whenever the probability measure $H_{\alpha} / d$ is absolutely continuous; the corresponding density $h_{\alpha} / d$ is then related to $v$ through (14). The latter is given by Coles and Tawn (1991, p. 381) as

$$
h_{\alpha}\left(w_{1}, \ldots, w_{d}\right)=\left\{\prod_{i=1}^{d-1}(i \alpha-1)\right\}\left(\prod_{i=1}^{d} w_{i}\right)^{-\alpha-1}\left(\sum_{i=1}^{d} w_{i}^{-\alpha}\right)^{1 / \alpha-d} .
$$

\section{CONNECTION WITH EARLIER WORK}

As mentioned in the Introduction, the work of Wüthrich (2003) and Alink et al. $(2004,2005)$ concerns the case where $D$ is Archimedean. Since they model their risks as random variables $Y_{1}, \ldots, Y_{d}$ taking negative values in case of a large aggregate loss $S^{\prime}=Y_{1}+\cdots+Y_{d}$, they are led to define

$$
\Delta=\lim _{t \rightarrow \infty} \frac{\mathrm{P}\left(S^{\prime} \leq-t\right)}{\mathrm{P}\left(Y_{i} \leq-t\right)} .
$$

The latter immediately reduces to (2) with $x=1$ upon setting $X_{i}=-Y_{i}$ for all $i \in\{1, \ldots, d\}$. The survival function of $X$ is then of the form (4) with $D$ given by (6). Using (5), one can thus see that the copula associated with $X$ is

$$
C\left(u_{1}, \ldots, u_{d}\right)=\sum_{I \subset\{1, \ldots, d\}}(-1)^{|I|} \phi^{-1}\left\{\sum_{i \in I} \phi\left(1-u_{i}\right)\right\},
$$

with the convention that an empty sum vanishes.

\subsection{Derivation of $\Delta$}

In order to compute $\Delta$, one must first determine $v_{\alpha}\left([0, x]^{c}\right)$ for arbitrary $x \in(0, \infty)^{d}$. Combining relations (15) and (19), one has 


$$
\begin{aligned}
v_{\alpha}\left([0, x]^{c}\right) & =\lim _{t \rightarrow \infty} t\left[1-\sum_{I \subset\{1, \ldots, d\}}(-1)^{|I|} \phi^{-1}\left\{\sum_{i \in I} \phi\left(\frac{1}{t x_{i}}\right)\right\}\right] \\
& =-\sum_{i=1}^{d} \frac{1}{x_{i}}+\sum_{|I| \geq 2}(-1)^{|I|} \lim _{t \rightarrow \infty} t \phi^{-1}\left\{\sum_{i \in I} \phi\left(\frac{1}{t x_{i}}\right)\right\} .
\end{aligned}
$$

Following Wüthrich (2003) and Alink et al. (2004, 2005), now suppose that $\phi(1 / t) \in \mathrm{RV}(\alpha)$ for some $\alpha>0$, whence $\phi^{-1}(t) \in \mathrm{RV}(-1 / \alpha)$. A standard regularvariation argument then yields

$$
\lim _{t \rightarrow \infty} t \phi^{-1}\left\{\sum_{i \in I} \phi\left(\frac{1}{t x_{i}}\right)\right\}=\left(\sum_{i \in I} x_{i}^{\alpha}\right)^{-1 / \alpha}
$$

Therefore,

$$
v_{\alpha}\left([0, x]^{c}\right)=-\sum_{I \subset\{1, \ldots, d\}}(-1)^{|I|}\left(\sum_{i \in I} x_{i}^{\alpha}\right)^{-1 / \alpha} .
$$

Calling on (14), one thus gets

$$
h_{\alpha}\left(w_{1}, \ldots, w_{d}\right)=\left\{\prod_{i=1}^{d-1}(i \alpha+1)\right\}\left(\prod_{i=1}^{d} w_{i}\right)^{\alpha-1}\left(\sum_{i=1}^{d} w_{i}^{\alpha}\right)^{-1 / \alpha-d} .
$$

As a consequence, an expression ensues for

$$
\Delta=\Delta_{2}(\alpha, \beta)=\int_{\mathbb{S}_{d-1}}\left(w_{1}^{1 / \beta}+\cdots+w_{d}^{1 / \beta}\right)^{\beta} h_{\alpha}\left(w_{1}, \ldots, w_{d}\right) d w_{1} \cdots d w_{d}
$$

which is equivalent to formula (2.3) of Alink et al. (2004). Note in passing that the extreme-value copula

$$
C_{\alpha}^{*}\left(u_{1}, \ldots, u_{d}\right)=\exp \left[\sum_{I \subset\{1, \ldots, d\}}(-1)^{|I|}\left\{\sum_{i \in I}\left|\log \left(u_{i}\right)\right|^{-\alpha}\right\}^{-1 / \alpha}\right]
$$

associated with $v_{\alpha}$ through (16) is a multivariate version of the so-called Galambos copula.

\subsection{Basic properties of $\Delta_{i}(\alpha, \beta)$ for $i=1,2$}

Theorem 2.5 of Alink et al. (2004) states basic properties of $\Delta_{2}(\alpha, \beta)$ in the special case $d=2$. Some of these facts, illustrated in Figure 1, extend to general $d \geq 2$ as well as to the constants $\Delta_{1}(\alpha, \beta)$ introduced in Section 3.4. 


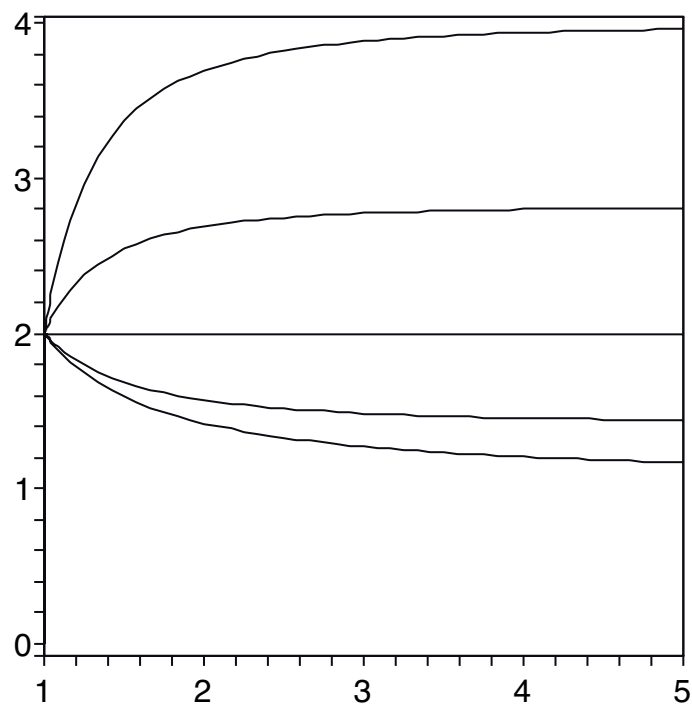

FIGURE 1: Graph of $\Delta_{1}(\alpha, \beta)$ as a function of $\alpha$ for different choices of $\beta$ when $d=2$; from top to bottom, $\beta=2,1.5,1,0.5,0.1$.

Proposition 4.1. Let $\Delta_{1}(\alpha, \beta)$ and $\Delta_{2}(\alpha, \beta)$ be defined by equations (18) and (20), respectively. For $i=1,2$, one has $\Delta_{i}(\alpha, \beta) \rightarrow \Delta_{i}(\alpha, 1)=d$ as $\beta \rightarrow 1$ and $\Delta_{i}(\alpha, \beta) \rightarrow d^{\beta}$ as $\alpha \rightarrow \infty$.

Proof. That $\Delta_{i}(\alpha, \beta) \rightarrow \Delta_{i}(\alpha, 1)=d$ as $\beta \rightarrow 1$ is a trivial consequence of the fact that $H_{\alpha}\left(\mathbb{S}_{d-1}\right)=d$. To establish the second limit, observe that since $v_{\alpha}\left([0, x]^{c}\right) \rightarrow$ $\max (1 / x)$ when $\alpha \rightarrow \infty$, it is immediate from (18) that the limiting spectral measure $H_{\infty}$ must concentrate all its mass, $d$, on the point $(1 / d, \ldots, 1 / d)$.

Remark 4.2. Alink et al. (2004) further show that if $d=2$, then $\Delta_{2}(\alpha, \beta)$ is either decreasing or increasing in $\alpha$, according as $\beta \leq 1$ or $\beta \geq 1$. Arguments similar to theirs can be used to check that the same is true of $\Delta_{1}(\alpha, \beta)$ also. In fact, it would appear that these monotonicity properties hold for arbitrary $d \geq 2$, but a proof remains elusive.

\section{DisCusSION}

This note has shown how results from multivariate extreme-value theory can be used to extend the work of Wüthrich (2003) and Alink et al. $(2004,2005)$ on the tail behavior of a sum $S=X_{1}+\cdots+X_{d}$ of dependent insurance claims. While these authors concentrated their analysis on exchangeable risks connected through an Archimedean copula, it was seen that their results readily extend to arbitrary dependence structures. 
For the sake of brevity, the presentation was limited here to the case where $X$ exhibits multivariate regular variation, which implies that its components belong to the domain of attraction of the Fréchet family of extreme-value distributions. However, simple adaptations are possible for Gumbel and Weibull extreme-value attractors.

The study of the parameter $\Delta$ initiated by Wüthrich (2003) and Alink et al. $(2004,2005)$ has an obvious interest from an actuarial or financial perspective, in that it allows for an approximation of very large quantiles of the distribution of the aggregate $S$ through those of the individual claims $X_{i}$ via $\mathrm{P}(S>t) \approx$ $\Delta \mathrm{P}\left(X_{i}>t\right)$. In terms of Value-at-Risk, for example, the implication is that for very small values of $p \in(0,1)$,

$$
\operatorname{VaR}_{p}(S)=\inf \{t \in \mathbb{R}: \mathrm{P}(S>t) \geq p\} \approx \operatorname{VaR}_{p / \Delta}\left(X_{i}\right) .
$$

This approximation would be handy, e.g., in situations where an estimate is required for $\operatorname{VaR}_{p}(S)$ but the analyst does not have enough data to circumscribe the dependence structure of the components of $X$. Under the assumption that the $X_{i}$ are identically distributed, an estimate of $\operatorname{VaR}_{p}\left(X_{1}\right)=\cdots=\operatorname{VaR}_{p}\left(X_{d}\right)$ would presumably be easier to obtain and, coupled with different choices of $\Delta$, this would yield at least a range of values for $\operatorname{VaR}_{p}(S)$. A simple expression for $\Delta$, such as (7), coupled with an understanding of the basic properties of this constant, would be advantageous in this context. See Alink et al. (2004) for a partial study of diversification effects using the VaR, and Alink et al. (2005) for parallel work on an alternative risk measure called expected shortfall.

Mathematically speaking, much remains to be done to understand the properties of the constant $\Delta$ under various structures of dependence. However, possibly the single most puzzling observation uncovered by the present investigation is the fact that for claims connected through an Archimedean family of copulas, the value of $\Delta$ is identically equal to $d$, irrespective of the strength of dependence, so long as $X_{i} \in \operatorname{RV}(-1)$ for every $i \in\{1, \ldots, d\}$. Further work will be required to delineate the exact circumstances under which this phenomenon occurs, not to speak of its meaning and impact in applications.

\section{ACKNOWLEDGMENTS}

This work was funded in part by the Natural Sciences and Engineering Research Council of Canada, by the Fonds québécois de la recherche sur la nature et les technologies, and by the Institut de finance mathématique de Montréal. The authors are grateful to the referees for comments that led to substantial improvement in the exposition.

\section{REFERENCES}

Alink, S., LöWE, M. and WÜTHRICH, M.V. (2004) Diversification of aggregate dependent risks. Insurance: Mathematics and Economics 35, 77-95. 
Alink, S., Löwe, M. and WÜThrich, M.V. (2005) Analysis of the expected shortfall of aggregate dependent risks. Astin Bulletin 35, 25-43.

Beirlant, J., Goegebeur, Y., Segers, J. and Teugels, J. (2004) Statistics of extremes: theory and applications. Wiley, New York.

CAPÉraÀ, P., Fougères, A.-L. and Genest, C. (2000) Bivariate distributions with given extreme value attractor. Journal of Multivariate Analysis 72, 30-49.

Cherubini, U., Luciano, E. and Vecchiato, W. (2004) Copula methods in finance. Wiley, New York.

Coles, S.G. and TAWn, J.A. (1991) Modelling extreme multivariate events. Journal of the Royal Statistical Society Series B 53, 377-392.

DE HAAN, L. and RESNICK, S.I. (1977) Limit theory for multidimensional sample extremes. Zeitschrift für Wahrscheinlichkeitstheorie und verwandte Gebiete 40, 317-337.

FELLER, W. (1971) An introduction to probability theory and its applications. Volume II, Second Edition, Wiley, New York.

Frees, E.W. and VAldeZ, E.A. (1998) Understanding relationships using copulas. North American Actuarial Journal 2, 1-25.

Genest, C. and MacKay, R.J. (1986) Copules archimédiennes et familles de lois bidimensionnelles dont les marges sont données. The Canadian Journal of Statistics 14, 145-159.

Genest, C. and Rivest, L.-P. (1989) A characterization of Gumbel's family of extreme value distributions. Statistics and Probability Letters 8, 207-211.

HuANG, X. (1992) Statistics of bivariate extremes. Doctoral dissertation, Tinbergen Institute Research series no 22, Erasmus Universiteit, Rotterdam, The Netherlands.

Joe, H. (1997) Multivariate models and dependence concepts. Chapman \& Hall, London.

Miкоsch, T. (2004) Modeling dependence and tails of financial time series. In Extreme Values in Finance, Telecommunications, and the Environment, (B. Finkelstädt and H. Rootzén, Eds.), Chapman \& Hall, London, pp. 185-286.

Nelsen, R.B. (1999) An introduction to copulas. Springer, New York.

PickAnds, J. (1981) Multivariate extreme value distributions. Proceedings, 43rd Session of the International Statistical Institute. Buenos Aires, Argentina, Book 2, pp. 859-878.

RESNICK, S.I. (1987) Extreme values, regular variation, and point processes. Springer, New York.

RESNICK, S.I. (2004) The extremal dependence measure and asymptotic independence. Stochastic Models 20, 205-227.

TAwn, J.A. (1988) Bivariate extreme value theory: Models and estimation. Biometrika 75, 397-415.

WÜTHRICH, M.V. (2003) Asymptotic value-at-risk estimates for sums of dependent random variables. Astin Bulletin 33, 75-92.

\section{PhilipPe BARbe}

Centre national de la recherche scientifique

90, rue de Vaugirard

75006 Paris, France

AnNe-Laure Fougères

Équipe Modal'X

Unité de formation et de recherche SEGMI

Université Paris $X$ - Nanterre

200, avenue de la République

92000 Nanterre, France

Christian Genest

Département de mathématiques et de statistique

Université Laval

Québec (Québec)

Canada G1K 7 P4 